\title{
Register nominum ad Vol. XXXI
}

Amram, S., v. Coelho, E. Arbeit, S. R., v. Moret, P. Arnovljevic, V., 31, 420 (S) Avril, P. B,, 31, $183(\mathrm{~B})$

Behn, H., v. Schaub, F. Bergmann, K., v. Fejfar, Z. Bilger, R., 31, 242 (S) Biörck, G., 31, 558

(S) Bischoff, L., 31, 343 (S) Bordalo o Sá, A., v. Coelho, ] Brod, J., 31, 500 (S) -, and Fencl, V., 31, 494 (S) Bühlmann, A., v. Schaub, F. Bu§u, I., v. Kleinermann, L.

Caruzzo, C, v. Magri, G.

Carvalho, Lopo de, v. Madeira-Pinto, P.

Coelho, E., Paduá, F. de, Amram, S., Bordalo o Sá, A., Serras Pereira, J., Maltez, J., Duarte, C.

A., Salez Luiz, A., et Maria, A. B., 31, 426 (S)

Cottier, P., Weller, J. M., und Hoobler, S. W., 31, 278 (S)

Cuénod, C.-L., v. Moret, P.

Dailheu-Geoffroy, P., 31, 551 (S) Desbaillets, P., Reymond, Cl., et Rivier, J.-L., 31, 307 (S)

Register nom

Desruelles, J., v. Merlen, J.-F. Duarte, C. A., v. Coelho, E. Duchosal, P. W., v. Moret, P.

Enescu, N., v. Kleinermann, L. Enyedi, C, v. Huttmann, A.

Fabre, J., 31, 219 (S)

Fejfar, Z., Bergmann, K., Fejfarová,

M., and Valach, A., 31, 461 (S) -, v. Widimský, J. Fejfarová, M. H., v. Fejfar, Z. -, v. Widimský,

J. Fencl, V., v. Brod, J. Ferrero, C, 31, 296 (S) Frumuzache, A., v. Huttmann, A.

Gamna, G., v. Magri, G. Gartner, W., und Schäfer, H., 31,

235 (S) Gençer, F., 31, 469 (S) Ghit,escu, T., v. Kleinermann, L. Gnüchtel, W., v. Holldack, K.

Gorelik, A. N., v. Lian, C. Grauer, H., 31, 86 Griesbach, R., und Kemper, F., 31,

182 (B) Gross, D., 31, 578 Gürtler, R., Lüthy, E., Senn, A., und

Søndergaard, T., 31, 313 (S) -, v. Lüthy, E.

Hackensellner, H. A., und Schmidt, K., 31, 162

Haefely, W., v. Schweizer, W.

Hartleb, O., v. Michel, D.

Hegglin, R., 31, 195 (S)

-, und Wiesmann, W., 31, 109

Hejl, Z., 31, 375 (S)

Herzog, H., v. Schweizer, W.

Hirt, H.-R., 31, 599

Holldack, K., Gnüchtel, W., und Stoffregen, J., 31, 176

-, v. Kuhn, E.

Holzmann, M., 31, 209 (S)

-, und Kieser, $\mathrm{Ch}, 31,36$

Hoobler, S. W., v. Cottier, P.

Horeman, H. W., v. Noordengraaf, A.

Hünnemeyer, F., v. Lüthy, E. 
Hürlimann, A., v. Imhof, P.

Huttmann, A., Enyedi, C., Frumuzache, A., Iancu, I., Mo7/8oiu, Gh., Stefẫnescu, C. R., Taffet, E., und Uláriu, I., 31, 536 (S)

ad Vol. XXXI 627

Iancu, I., v. Huttmann, A. Imhof, P., Hürlimann, A., und Steinmann, B., 31, 272 (S)

Jacobi, M., v. Lian, C. Jacovella, G., v. Mussafia, A. Jornod, J., Moret, P., et Stamm, O.,

31, 237 (S)

Karlagener, M., 31, 79 Kaufmann, E., und Staemmler, M.,

31, 184 (B) Kemper, F., v. Griesbach, R. Kieser, Ch., v. Holzmann, M. Kirchhoff, H. W., v.

Musshoff, K. Kisch, B., 31, 344 (B) Kleinermann, L., Ghitescu, T., Bu $3 / 8 u$,

I., Enescu, N., und Lupu, A., 31,

475 (S) Klepzig, II., v. Musshoff, K. Köhler, U., 31, 512 (S) Korth, C., 31, 182 (B) Krautwald, A., und Stecher, W., 31,

523 (S) Kuhlmann, F., 31, 566 (B) Kuhn, E., Holldack, K., und

Winckler, H., 31, 567

Lian, C., Gorelik, A. N., et Jacobi,

M., 31, 180 (B) Luiz, A. S., v. Coelho, E. Lupu, A., v. Kleinermann, L. Lüthy, E., Gürtler, R., Hünnemeyer,

F., und Schmid, F., 31, 252 (S) -, und Stucki, P., 31, 5 -, v. Gürtler, R.

Madeira-Pinto, P., Ayres de Sousa,

and Lopo de Carvalho, 31, 401 (S) Magri, G., Caruzzo, G., Gamna, G.,

and Oddone, I., 31, 407 (S) Mahaim, Ch., et Nieuwenhuizen,

C. L. C. van, 31, 325 (S) Maier, C, und Spühler, H., 31,325 (S) Maltez, J., v. Coelho, E. Maria, A. B., v. Coelho, E. Matejicek, E., Takác, M., and

Stubnová, G., 31, 589 Maurea, C, Nylin, G., and Soll-

berger, A., 31, 350 (S) Meda, A., and Scansetti, G., 31, 144 Merlen, J.-F., et Desruelles, J., 31, 620 (B)

628 Register nominum

Michel, D., Rechenberger, J., und

HaГtleb, O., 31, 484 (S) Moret, P., Arbeit, S. R., Richmond,

R., and Schwartz, M. L., 31, 123 -, Cuénod, C.-L., et Duchosal, P. W.,

31, 258 (S) -, v. Jornod, J. Moßoiu, Gh., v. Huttmann, A. Mussafia, A., and Jacovella, G., 31,

173 Musshoff, K., Reindell, H., Klepzig,

H., und Kirchhoff, H. W., 31, 359

(S)

Nieuwenhuizen, C. L. C. van, v.

Mahaim, Ch. Noordengraaf, A., and Horeman,

H. W., 31, 416 (S) Nylin, G., v. Maurea, C.

Oddone, I., v. Magri, G. Oedman, P., v. Porjé, I. G.

Paduá, F. de, v. Coelho, E. Pereira, J. S., v. Coelho, E. Philipsson, J., v. Porjé, I. G. Porjé, I. G., Philipsson, J., and

Oedman, P., 31, 508 (S) -, and Rudewald, B., 31, 389 (S)

Rechenberger, J., v. Michel, D. Reindell, H., v. Musshoff, K. Reymond, Cl., v. Desbaillets, P.

Richmond, R., v. Moret, P. Rivier, J.-L., v. Desbaillets, P. Rudewald, B., v. Porjé, I. G.

Sá, A. B., v. Coelho, E. Scansetti, G., v. Meda, A. Schäfer, H., v. Gartner, W.

ad Vol. XXXI 
Schaub, F., Bühlmann, A., und

Behn, H., 31, 289 (S) Scheu, H., v. Spühler, O. Schmid, F., v. Lüthy, E. Schmidt, K., v.

Hackensellner, H. A. Scholer, H., 31, 331 (S) Schwartz, M. L., v. Moret, P. Schweizer, W.,

Herzog, H., und

Haefely, W., 31, 301 (S) Senn, A., v. Gürtler, R. Sollberger, A., v. Maurea, C. Søndergaard, T., v. Gürtler, R. Sousa, A. de, v. Madeira-Pinto, P. Spühler, H., v. Maier, C. Spühler, 0., und Scheu, H., 31, 51 Staemmler, M., v. Kaufmann, E. Stamm, O., v. Jornod, J. Stecher, W., v. Krautwald, A. §tefänescu, C. R., v. Huttmann, A. Steinmann, B., v. Imhof, P. Stoffregen, J., v. Holldack, K. Stubnová, G., v. Matejiéek, E. Stucki, P., v. Lüthy, E.

Taffet, E., v. Huttmann, A. Takác, M., v. Matejicek, E. Temesvári, A., 31, 572

Uláriu, I., v. Huttmann, A. Unghváry, L. 31, 139

Valach, A., v. Fejfar, Z.

Weidmann, S., 31, 186 (S) Weller, J. M., v. Cottier, P. Widimský, J., Fejfarová, M. H., and Fejfar, Z., 31, 381 (S) Wiesmann, W., v. Hegglin, R. Winckler, H., v. Kuhn, E. 